\title{
Editorial
}

\section{New Developments in Reproductive and Stress Neuroendocrinology}

\author{
Vincent Prevot ${ }^{a}$ Robert P. Millar ${ }^{b}$ \\ a Laboratory of Development and Plasticity of the Neuroendocrine Brain, INSERM, University of Lille, UMR-S 1172 , \\ Lille, France; ${ }^{\mathrm{b} C}$ Centre for Neuroendocrinology, Department of Immunology, University of Pretoria, Pretoria, South \\ Africa
}

In July 2018, Toronto hosted the 10th International Congress of Neuroendocrinology (ICN) and a number of the presenters were invited to submit their presentations for publication in a special issue of Neuroendocrinology, focusing on cutting edge developments in the neuroendocrine control of reproduction and stress. We are honored and delighted to present to you 10 superb articles addressing this topic.

Neuroendocrine regulation of fertility is governed by a complex neural network, primarily located in the hypothalamus, that converges onto $\mathrm{GnRH}$-producing neurons - the master regulators of gonadotropin secretion and postnatal gonadal growth and function. The orderly development of GnRH neurons, GnRH gene expression, and GnRH signaling is essential for sexual maturation and the healthy functioning of the hypothalamic-pituitary-gonadal (HPG) axis in mammals.

GnRH neurons are unusual neuroendocrine neurons, in that, unlike other paraventricular neurosecretory neurons that arise from the neuroepithelium of the third ventricle, they originate from the olfactory placode and migrate from the nose to the brain during embryonic development. In this issue, Oleari et al. [1] detail the increasingly recognized contribution of semaphorins to this event, as they are involved in axonal pathfinding, for the migration of GnRH neurons, while Hoffmann et al.
[2] discuss the roles played by three homeodomain proteins, Six 3, Vax 1, and Otx2, in the development and function of GnRH neurons.

At birth, GnRH neurons have reached their final destination within the hypothalamus. However, during postnatal development, GnRH neurons are subjected to a sequence of complex maturational events which ultimately lead to the acquisition of reproductive competence. Epigenetics has emerged as one of these and has been implicated as a significant regulatory mechanism of GnRH neuronal network postnatal activity, which is required for pubertal development and progression, as elaborated by Aylwin et al. [3].

The maintenance of fertility during adulthood requires continuous interactive dialogue between the hypothalamus, the pituitary, and the gonads. The steroid and peptide hormones released into the circulation exert positive and negative feedback actions at the different levels of the HPG axis. Barbotin et al. [4] intriguingly report on a novel role for the ovarian anti-Müllerian hormone in hypothalamic-pituitary function and suggest that it may play a role in the pathophysiology of polycystic ovary syndrome, which is one of the most common reproductive disorders currently affecting women.

Within the GnRH neuronal network, kisspeptin neurons, many of which also synthetize neurokinin B in the

\section{KARGER}

() 2019 (c) 2019 S. Karger AG, Basel
Prof. Vincent Prevot

Jean-Pierre Aubert Research Center

INSERM, U1172, CHU Lille

FR-59045 Lille Cedex (France)

E-Mail vincent.prevot@inserm.fr 
infundibular nucleus/arcuate nucleus, are a key player in the regulation of reproductive function. In this issue, Hrabovszky et al. [5] discuss sexually dimorphic agingrelated anatomical alterations of the kisspeptin and neurokinin B systems in humans. Altered neurokinin B signaling in postmenopausal women is thought to play a role in the advent of hot flushes. Modi and Dhillo [6] have conducted clinical studies in postmenopausal women and demonstrated that antagonists at the neurokinin $\mathrm{B}$ receptor, NK3R, are effective in reducing the incidence and severity of hot flushes.

In addition to their classical effects, ovarian hormones, and particularly gonadal steroids, exert organizational effects during development, the neonatal period being a particularly sensitive time window. This might reflect sex differences in the lifelong health consequences of adverse conditions at birth, such as hypoxia-ischemia, as elaborated by Zafer et al. [7] in this issue.

Stress or iatrogenic exposure to glucocorticoids during prenatal life may also have programming effects and result in affective and neuropsychiatric disorders in adults, in addition to causing lifelong alterations in function of the HPG axis. This is discussed by Shearer et al. [8] in this special issue, whereas Viho et al. [9] elaborate on the fact that selective glucocorticoid receptor modulators can be employed to selectively activate subsets of glucocorticoid receptor-coregulator interactions. These provide novel tools to identify molecular processes that are responsible for adaptive and maladaptive effects of glucocorticoids in the brain.

Finally, Jeanneteau et al. [10] discuss the fact that behavioral choices made by the brain during stress depend on the crosstalk between glucocorticoid and brain-derived neurotrophic factor (BDNF) signaling pathways acting in concert in the mesolimbic and corticolimbic neuronal networks controlling reward and emotion, respectively. BDNF signaling conditions glucocorticoid responses impacting on neural plasticity in the mesolimbic system.

We believe that the articles in this special issue will become seminal references for scientists working in the exciting field of the neuroendocrinology of stress and reproduction, and that it will also attract the interest of other neuroscientists not as yet involved in this fascinating area of research.

\section{References}

1 Oleari R, Lettieri A, Paganoni A, Zanieri L, Cariboni A. Semaphorin signaling in GnRH neurons: from development to disease. Neuroendocrinology. 2018. Available from: https://doi.org/10.1159/000495916.

2 Hoffmann H, Pandolfi E, Larder R, Mellon P. Haploinsufficiency of homeodomain proteins Six3, Vax1, and Otx2, causes subfertility in mice via distinct mechanisms. Neuroendocrinology. 2018.2 https://doi. org/10.1159/000494086.

3 Aylwin CF, Vigh-Conrad K, Lomniczi A. The emerging role of chromatin remodeling factors in female pubertal development. Neuroendocrinology. 2019. Available from: https:// doi.org/10.1159/000497745.
4 Barbotin AL, Peigné M, Malone SA, Giacobini P. Emerging Roles of Anti-Müllerian Hormone in Hypothalamic-Pituitary Function. Neuroendocrinology. 2019. Available from: https://doi.org/10.1159/000500689.

5 Hrabovszky E, Takács S, Göcz B, Skrapits K. New perspectives for anatomical and molecular studies of kisspeptin neurons in the aging human brain. Neuroendocrinology. 2019. Available from: https://doi. org/10.1159/000496566.

6 Modi M, Dhillo WS. Neurokinin 3 receptor antagonism: a novel treatment for menopausal hot flushes. Neuroendocrinology. 2018 Available from: https://doi. org/10.1159/000495889.

7 Zafer D, Aycan N, Ozaydin B, Kemanli P, Ferrazzano $\mathrm{P}$, Levine JE, et al. Sex differences in hippocampal memory and learning following neonatal brain injury: is there a role for $\mathrm{ER} \alpha$ ? Neuroendocrinology. 2019. Available from: https://doi.org/10.1159/000499661.
8 Shearer FJ, Wyrwoll CS, Holmes MC. The role of 11ß-hydroxy steroid dehydrogenase type 2 in glucocorticoid programming of affective and cognitive behaviours. Neuroendocrinology. 2019. Available from: https://doi. org/10.1159/000499660.

9 Viho EM, Buurstede JC, Mahfouz A, Koorneef LL, van Weert LT, Houtman R, et al. Corticosteroid action in the brain: the potential of selective receptor modulation. Neuroendocrinology. 2019. Available from: https://doi. org/10.1159/000499659.

10 Jeanneteau F, Borie A, Chao MV, Garabedian MJ. Bridging the gap between brain-derived neurotrophic factor and glucocorticoid effects on brain networks. Neuroendocrinology. 2018. Available from: https://doi. org/10.1159/000496392. 